Coastal Dynamics 2009

Paper No.

\title{
SEDIMENT TRANSPORT MODULE FOR A B-GRID COASTAL SHELF OCEAN MODEL
}

\author{
Laurent O. Amoudry ${ }^{1}$, Alejandro J. Souza ${ }^{2}$ and Jason T. Holt ${ }^{3}$
}

\begin{abstract}
We present a three-dimensional sediment transport model implemented within the Proudman Oceanographic Laboratory Coastal Ocean System (POLCOMS). An unlimited number of sediment classes can be transported both as suspended load and bed load. Suspended sediment concentration is calculated following advection-diffusion schemes similar to those used for other scalar quantities. The location of the sediment bed is obtained from near-bed sediment mass conservation and the sediment bed is represented in the model by a layered structure. The ability to reproduce suspended concentration profiles and morphological evolution for a simple case is tested against laboratory experiments of trench migration in a flume
\end{abstract}

Key words: sediment transport, morphodynamics, bed load, numerical modelling,

\section{Introduction}

Modelling systems able to predict the evolution of coastal zones have now become essential to engineering and policy making communities. Sediment transport is a key component in coastal interactions, and models describing the fate of particles and morphodynamics in coastal and estuarine environments have grown into vital tools.

To be able to correctly reproduce and predict sediment dynamics, the full three-dimensional structure of the flow has to be described in many complex situations, in particular depth-averaged models fail to represent adequately cases for which buoyancy stratification is significant. Fully three dimensional models that predict suspended sediment concentrations, bed load transport rates, and two-dimensional bathymetric evolution, are now being developed. To date, such models (e.g., Lesser et al., 2004; Warner et al., 2008) tend to be mostly using C-grids. B-grid models such as the Proudman Oceanographic Laboratory Coastal Ocean Modelling System (POLCOMS) (e.g., Holt and James, 2001) are traditionally considered to help prevent numerical dispersion of frontal velocity features.

We will discuss here the implementation of suspended sediment transport, bed load transport and morphodynamical changes within the POLCOMS. Such an effort will provide great capabilities and flexibility to the overall modelling system by building on pre-existing synergies. POLCOMS has indeed already been coupled to a wave model (Osuna et al., 2004), the General Ocean Turbulence Model (GOTM) (Holt and Umlauf, 2008) and an ecosystem model (Allen et al, 2001). In spite of the grid specificity of POLCOMS, the sediment algorithms will incorporate approaches from other similar finite difference sediment transport models (e.g., Lesser et al., 2004; Warner et al., 2008). Because of the close interaction between the hydrodynamics and the sediment dynamics, the new algorithms are implemented directly within the hydrodynamic model in the POLCOMS.

We will first briefly summarize the existing hydrodynamic model in the POLCOMS, before describing in details the sediment transport model. We will then discuss some aspects of the numerical schemes employed. Finally, we present the comparison of model results with laboratory experimental data of trench migration for which both the velocity and suspended sediment concentration (SSC) vertical profiles and the morphological evolution are well predicted by the sediment transport model implemented in the POLCOMS.

1 Proudman Oceanographic Laboratory, 6 Bownlow Street, Liverpool, L3 5DA, UK. laou@pol.ac.uk 2 Proudman Oceanographic Laboratory, 6 Bownlow Street, Liverpool, L3 5DA, UK. ajso@pol.ac.uk 3 Proudman Oceanographic Laboratory, 6 Bownlow Street, Liverpool, L3 5DA, UK. jholt@pol.ac.uk 
Coastal Dynamics 2009

Paper No.

\section{Model description}

\subsection{Hydrodynamic model}

The physical model is based on a three-dimensional baroclinic model and solves the incompressible, hydrostatic, Boussinesq type shallow water equations of motions. The governing equations can be expressed and solved using either Cartesian or spherical polar coordinates. A time splitting technique is employed to calculate the barotropic and baroclinic components. Following this approach, flow velocities are separated into depth averaged and depth varying components. Equations of motions for both the depth averaged and depth-varying parts are then derived and solved numerically (see Holt and James (2001) for more details on these equations). The free surface location is obtained following convergence or divergence of the water fluxes over the entire water depth and scalars such as temperature and salinity follow a classical advection-diffusion governing equation.

In the hydrodynamic model, the turbulent stresses and fluxes in the depth varying equations are replaced following respectively the turbulent viscosity and gradient diffusion hypotheses. This results in diffusive terms that necessitate further closure using a turbulence model. Horizontal diffusivities are neglected and the vertical diffusivities are obtained either by a one-equation turbulence model or by coupling the hydrodynamic model to the General Ocean Turbulence Model (GOTM, see www.gotm.net). When coupling POLCOMS with GOTM, several turbulence models are available and the Canuto et al. (2001) model is selected by default in the present study. This turbulence closure can be seen as a k- $\varepsilon$ model for which the stability function (i.e., the $\mathrm{C}_{\mu}$ constant) results from an Algebraic Reynolds Stress Model under the boundary layer assumption. The Prandtl number is neither specified as a constant nor follows empirical relationships, but is a consequence of the model itself and is a function of the shear and of the buoyancy.

\subsection{Sediment transport model}

The sediment transport model consists of three interconnected modules respectively calculating suspended load, bed load, and bed morphodynamics. Even though bed shear stress calculations are also part of the hydrodynamic model, the methods used are discussed in this section due to their strong impact on sediment transport. Because of the multiple interactions between the hydrodynamic model and the sediment modules, the three modules have been implemented within the hydrodynamic model.

An infinite number of sediment classes can be represented, each of which is described by user-defined values for the sediment grain diameter $D$, the dry sediment density $\rho_{s}$, the critical erosion bed shear

stress $\tau_{c e}$ and an erodibility constant $E_{0}$. The sediment bed consists of a constant user-defined number of layers, each of which is described by spatially and time varying thicknesses, sediment class distributions, porosities and ages.

\subsection{1. $\quad$ Bed shear stress}

Bed shear stress predictions are critical to sediment transport formulations. Different algorithms are implemented here depending on the flow conditions. For currents (velocities varying on a time scale much longer than the time step), the bottom shear stress is calculated using a drag coefficient formula based on the near-bed velocity following a rough wall logarithmic profile:

$$
\left(F_{B}, G_{B}\right)=\left(u_{B}, v_{B}\right) \frac{\kappa^{2}}{\ln \left(\delta_{0} / z_{0}\right)} \sqrt{u_{B}^{2}+v_{B}^{2}}
$$

where $F_{B}$ and $G_{B}$ are the two components of the bed shear stress, $u_{B}$ and $v_{B}$ the two components of the near bed velocity defined at an elevation $\delta_{0}$ above the sea bed. $z_{0}$ is the bed roughness and $\kappa=0.41$ the von Karman constant. 
Coastal Dynamics 2009

Paper No.

For wave-current interactions, the mean combined $\tau_{m}$, maximum combined $\tau_{\max }$ and wave bed shear stresses $\tau_{w}$ are all calculated. The mean combined stress is then projected on the horizontal grid to obtain $F_{B}$ and $G_{B}$ used in the hydrodynamic calculations. Both an iterative solution following the model of Madsen (1994) and the explicit formulas of Soulsby (1995) are implemented in POLCOMS.

The bed roughness can either be fixed as a user defined constant or can be calculated within the numerical model. In the latter case, it is considered to be the sum of the grain roughness, the bed load roughness and the roughness due to sub-grid scale bed forms (ripples). The grain roughness is chosen to be proportional to the bed surface mean sediment diameter, the bed load roughness follows the formulation of Wiberg and Rubin (1989) and the bed form roughness is taken to be a function of ripple length and height (Grant and Madsen, 1982). The total bed roughness is then given as

$$
z_{0}=\alpha_{g} D_{50}+\alpha_{b l} \frac{a_{1} \tau_{\max } / \tau_{c r}}{1+a_{2} \tau_{\max } / \tau_{c r}} D_{50}+\alpha_{r} \frac{\eta^{2}}{\lambda}
$$

where the right hand side terms represent respectively the grain roughness, the bed load roughness and the bed form roughness. $D_{50}$ is the median sediment diameter at the sediment bed (taking into account all sediment classes). The coefficient $a_{2}$ is taken to be a function of the grain diameter (Wiberg and Rubin, 1989):

$$
a_{2}=0.0204\left[\ln \left(100 D_{50}\right)\right]^{2}+0.0220 \ln \left(100 D_{50}\right)+0.0709
$$

$\tau_{c r}$ is the critical stress for initiation of motion and the remaining coefficients involved in the determination of the roughness are summarized in table 1 . The value for $\alpha_{r}$ varies in the literature (e.g., 0.267 from Nielsen, 1992; about 0.92 from Grant and Madsen, 1982) and has thus been left as a user input to be chosen within the range indicated in table 1. Finally, the ripple height $\eta$ and the ripple length $\lambda$ are calculated from the value of the wave orbital diameter using the Malarkey and Davies (2003) explicit implementation of the Wiberg and Harris (1994) ripple predictor.

Table 1: Summary of coefficients in bed roughness determination.

\begin{tabular}{|l|l|}
\hline Coefficient & Value \\
\hline$\alpha_{g}$ & $2.5 / 30$ \\
\hline$\alpha_{b l}$ & 0.056 \\
\hline$a_{1}$ & 0.68 \\
\hline$\alpha_{r}$ & 0.1 to 1 \\
\hline
\end{tabular}

\subsubsection{Suspended load}

The suspended sediment transport model solves an advection-diffusion equation for each sediment class:

$$
\frac{\partial c}{\partial t}=-L(c)+D(c)+W_{s} \frac{\partial c}{\partial z}+S_{c}
$$

where $S_{c}$ is a sediment source/sink term. The exchange with the bed due to gravitational settling and erosion is included as a source/sink term for the bottommost grid of the water column. Deposition is taken to occur at all bed shear stresses and erosion is modelled following a linear dependence to an excess shear stress: 
Coastal Dynamics 2009

Paper No.

$$
E=E_{0}(1-\varphi)\left(\frac{\tau_{\max }}{\tau_{c e}}-1\right)
$$

where $E_{0}$ is the used-defined erodibility constant and $\varphi$ is the top bed layer porosity. $\tau_{\max }$ is the combined wave-current maximum shear stress and $\tau_{c e}$ is the user-defined critical erosion stress.

For each sediment class, the settling velocity is computed from the sediment diameter, the sediment specific gravity and the water kinematic viscosity coefficient following van Rijn (1993):

$$
W_{s}=\left\{\begin{array}{ccc}
\frac{(s-1) g D^{2}}{18 v} & \text { for } & 1<D \leq 100 \mu m \\
\frac{10 v}{D}\left[\left(1+\frac{0.01(s-1) g D^{3}}{v^{2}}\right)^{1 / 2}-1\right] & \text { for } & 100<D<1000 \mu m \\
1.1[(s-1) g D]^{1 / 2} & \text { for } & D \geq 1000 \mu m
\end{array}\right.
$$

Both the advective term and the diffusive term are calculated following the same approaches as for other scalars (temperature and salinity). Furthermore, the sediment Schmidt number (ratio of the turbulent eddy viscosity by the sediment turbulent diffusivity) is taken to be identical to the buoyancy Prandtl number.

\subsubsection{Bed load}

Bed load is typically related to the bed shear stresses via non-dimensional equations, in which the nondimensional bed load transport rate is

$$
\Phi_{B}=\frac{Q_{B}}{\rho_{s} \sqrt{(s-1) g D^{3}}},
$$

and the non-dimensional bed shear stresses are

$$
\left(\theta_{\max }, \theta_{m}, \theta_{w}\right)=\frac{\left(\tau_{\max }, \tau_{m}, \tau_{w}\right)}{(s-1) g D}
$$

Two bed load formulations have been implemented in POLCOMS. The Meyer-Peter and Muller (1948) formula for currents gives the magnitude of the non-dimensional bed load transport $\Phi_{B}$ as

$$
\Phi_{B}=8(\theta-0.047)^{3 / 2}
$$

The Soulsby and Damgaard (2005) formulation is also implemented for currents or wave-current interactions and provides equations for the bed load transport parallel to the current direction:

$$
\Phi_{c}=\max \left(\Phi_{c 1}, \Phi_{c 2}\right)
$$

with 
Coastal Dynamics 2009

Paper No.

$$
\begin{aligned}
& \Phi_{c 1}=12 \theta_{m}^{1 / 2}\left(\theta_{m}-\theta_{c r}\right) \\
& \Phi_{c 2}=12(0.9534+0.1907 \cos 2 \phi) \theta_{w}^{1 / 2} \theta_{m}+12\left(0.229 \Delta_{w} \theta_{w}^{3 / 2} \cos \phi\right)
\end{aligned}
$$

The bed load transport perpendicular to the current direction is given by

$$
\Phi_{n c}=12 \frac{0.1907 \theta_{w}^{2}}{\theta_{w}^{3 / 2}+1.5 \theta_{m}^{3 / 2}}\left(\theta_{m} \sin 2 \phi+1.2 \Delta_{w} \theta_{w} \sin \phi\right),
$$

where $\theta_{m}$ and $\theta_{w}$ respectively correspond to the mean and wave bed shear stresses. $\theta_{c r}$ is the critical Shields parameter for initiation of motion. $\phi$ is the angle between the current and the wave propagation directions, and $\Delta_{w}$ is the wave asymmetry factor (ratio of the amplitude of the second harmonic and the amplitude of the first harmonic). The bed load transport is then projected on to the horizontal grid and a multiplicative factor is applied to account for the local slope effects following Lesser et al. (2004):

$$
a_{s l}=\frac{\tan \varphi_{m}}{\left(\tan \varphi_{m}-\tan \beta\right) \cos \beta}
$$

where $\varphi_{m}$ is the friction angle of sediment and $\beta$ the local bed slope.

\subsubsection{Bed morphology}

The bed morphology module follows the approaches implemented in Harris and Wiberg (2001) and Warner et al. (2008). It performs both bed statigraphy (updating the sediment bed structure) and bed morphology (updating the location of the sediment bed) procedures.

The bed statigraphy first calculates an active layer thickness following Harris and Wiberg (1997):

$$
\delta_{a}=\max \left[0.007 \rho_{0}\left(\tau_{\max }-\overline{\tau_{c e}}\right), 0\right]+6 \mathrm{D}_{50}
$$

where the overbar denotes an average over all sediment classes. The thickness of the top layer is then enforced to be at least the active layer thickness. If necessary, the bed statigraphy is rearranged so that (i) the thickness of the top layer is equal to $\delta_{a}$, (ii) sediment mass is conserved, (iii) the number of bed layers is constant.

The bed morphology updates both the location of the sediment bed and the top layer thickness following the conservation of sediment mass between the bed, erosion, deposition, and bed load transport. A morphological factor can be applied to the various sediment fluxes, but total fluxes of sediment out of the bed are limited by the true mass of sediment available in the top layer. The top layer thickness is also limited by a user-defined upper threshold and, if necessary, a new layer is created and the bottom two layers merged.

The implementation of the bed morphology separates the top layer thickness calculations from the location of the sediment bed updating scheme. At each time step, a succession of bed statigraphy, top layer thickness calculations and bed statigraphy again is performed in the sediment transport module. The location of the sediment bed is updated simultaneously with the depth-averaged velocities to enforce conservation of fluid mass. Finally, the bottom boundary condition for the vertical velocity is set as the rate of change of the sea floor. 
Coastal Dynamics 2009

Paper No.

\section{Numerical solution}

\subsection{Grid}

POLCOMS uses a B-grid in the horizontal plane and a staggered approach for the vertical direction. In the horizontal plane, both velocity components are then defined at u-points, which are the centers of grid cells, half a grid box to the southwest of b-points, at which all scalars are defined (see figure 1). Sea-land boundaries can lie either at b-points or at u-points. In the first case, a free-slip condition is implemented while in the second case both free-slip or non-slip conditions can be chosen. Open boundaries always lie along b-points. The vertical discretization defines state variables (horizontal velocities and scalars) half a grid above and below the sea surface and sea bottom, while flux variables (e.g., vertical velocity) are defined at the sea bed and at the sea surface.

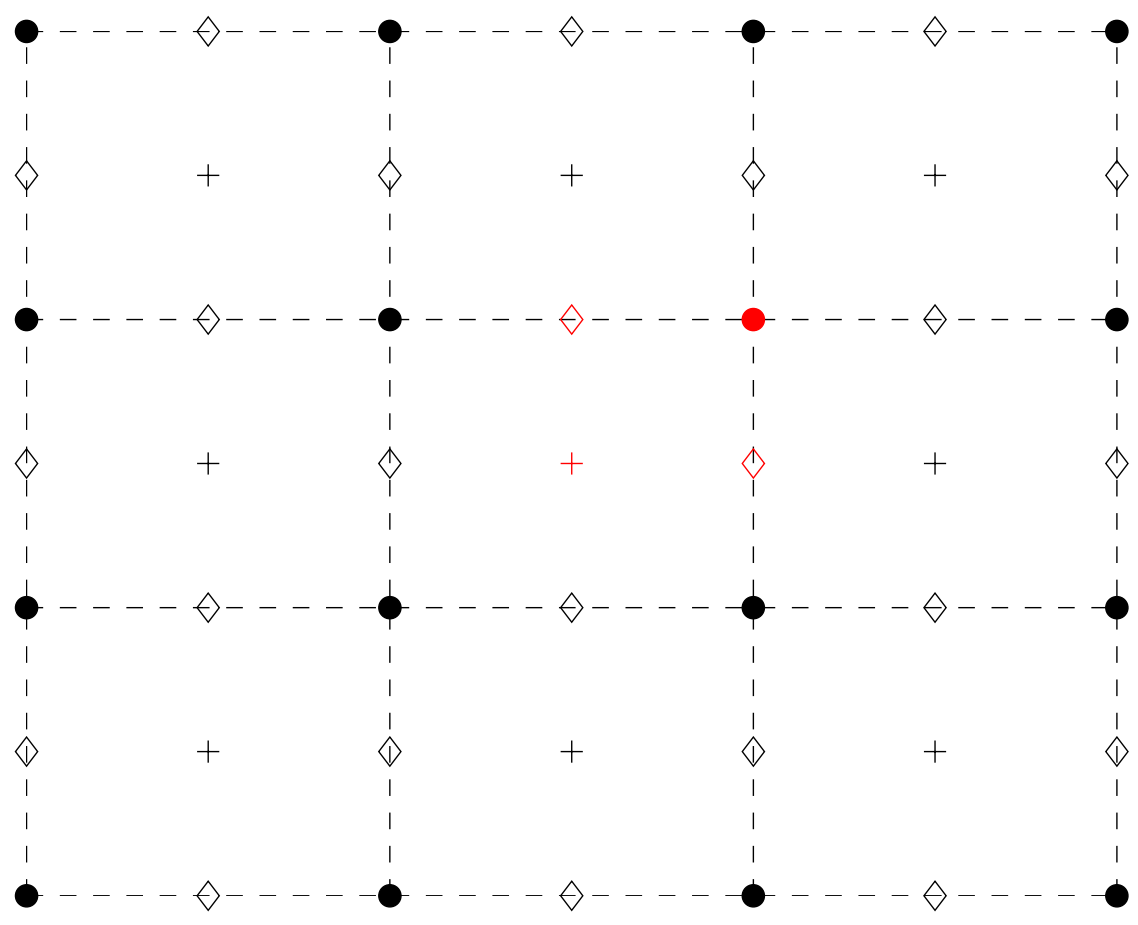

Figure 1. Representation of a B-grid. The u-points are located at the plus signs, the b-points (for scalars) are located at the black circles. The diamonds correspond to the location of the translated bed load fluxes. The red symbols correspond to the middle cell.

\subsection{Numerical schemes}

Details of the numerical schemes for the hydrodynamic model are given in Holt and James (2001) and are not repeated here. A forward time centred space technique is employed to solve the hydrodynamic governing equations. To prevent the B-grid scale noise, the free surface elevations are filtered following Killworth et al. (1991). Advective terms are calculated following a Piecewise Parabolic Method scheme (James, 1996) while the diffusive terms are computed following a fully implicit scheme.

The "sediment module" performs successively bed load calculations, vertical settling with a Piecewise Parabolic Method scheme, the exchange between the bed and the flow, the bed statigraphy, and finally the 
Coastal Dynamics 2009

Paper No.

advection and diffusion. The location of the sea bed is updated within the hydrodynamic model, simultaneously with the depth averaged velocities and free surface location.

Sediment variables are mostly defined at b-points (to the exception of the output bed load transport rate, bed shear stresses and bed roughnesses). Bed load transport rates are first calculated at the b-points from the interpolated values of the bed shear stress, and are then translated in the horizontal grid to the locations represented by diamonds in figure 1 to facilitate the bed evolution calculations at b-points which follows mass conservation.

\section{Model-data comparison on trench migration experiment}

We use the trench migration experiment reported in van Rijn (1987) to test the ability of the model to reproduce suspended sediment concentration (SSC) profiles and morphological evolutions. The setup consists of a 30 meter long, and $0.5 \mathrm{~m}$ wide straight channel in which a $0.2 \mathrm{~m}$ thick bed of well-sorted sediment of median diameter $160 \mu \mathrm{m}$ is installed. Three different trenches were excavated and their migration downstream was observed after 15 hours. The upstream flow is steady with a depth-averaged velocity of $0.51 \mathrm{~m} / \mathrm{s}$ and a depth of $0.39 \mathrm{~m}$.

The channel is discretised using a $0.1 \mathrm{~m}$ resolution in both horizontal directions and 20 constant $\sigma$ levels vertically. Implementing variable $\sigma$ levels was not found to change the numerical solution in the present case. The channel bed slope and a constant bed roughness are determined so that the flow rate remains uniform along the test section. Both the flow velocity and suspended concentration are then allowed to reach an "initial" steady state solution. This solution is then used as starting point for the morphodynamic computation, which employs a morphological factor of 10 due to the small time step enforced. The physical and numerical parameters are summarized in table 1 . The critical erosion stress is chosen to be about equal to the critical stress for initiation of motion for the specific sediment used, as estimated from the Shields curve. The erodibility is then chosen based on model-data comparisons of the suspended concentration initial profiles.

Table 2: Model parameters for trench migration numerical simulations.

\begin{tabular}{|l|c|}
\hline Model parameter & Value \\
\hline Median sediment diameter & $0.16 \mathrm{~mm}$ \\
\hline Sediment dry density & $2650 \mathrm{~kg} / \mathrm{m}^{3}$ \\
\hline Critical erosion stress & $0.00017 \mathrm{~m}^{2} / \mathrm{s}^{2}$ \\
\hline Erodibility constant & $0.012 \mathrm{~kg} / \mathrm{m}^{2} / \mathrm{s}$ \\
\hline Bed porosity & 0.4 \\
\hline Bed roughness & $0.0008 \mathrm{~m}$ \\
\hline Bed slope & 0.00042 \\
\hline Upstream depth averaged velocity & $0.51 \mathrm{~m} / \mathrm{s}$ \\
\hline Time step & $0.01 \mathrm{~s}$ \\
\hline Morphology factor & 10 \\
\hline
\end{tabular}

Model data comparisons for the "initial" steady state solution and the trench migration are presented in figure 1, where the top panel exhibits the flow velocity and concentration profiles at five locations along the trench and the bottom panel illustrates the trench migration after 15 hours. A numerical simulation using a morphological factor of 1 was performed to verify the lack of impact of this parameter. In spite of slight under-predictions of the flow velocity in the excavated trench region, the initial profiles are well predicted at the five locations. The trench migration and the final trench depth are both slightly overpredicted by the numerical model. 

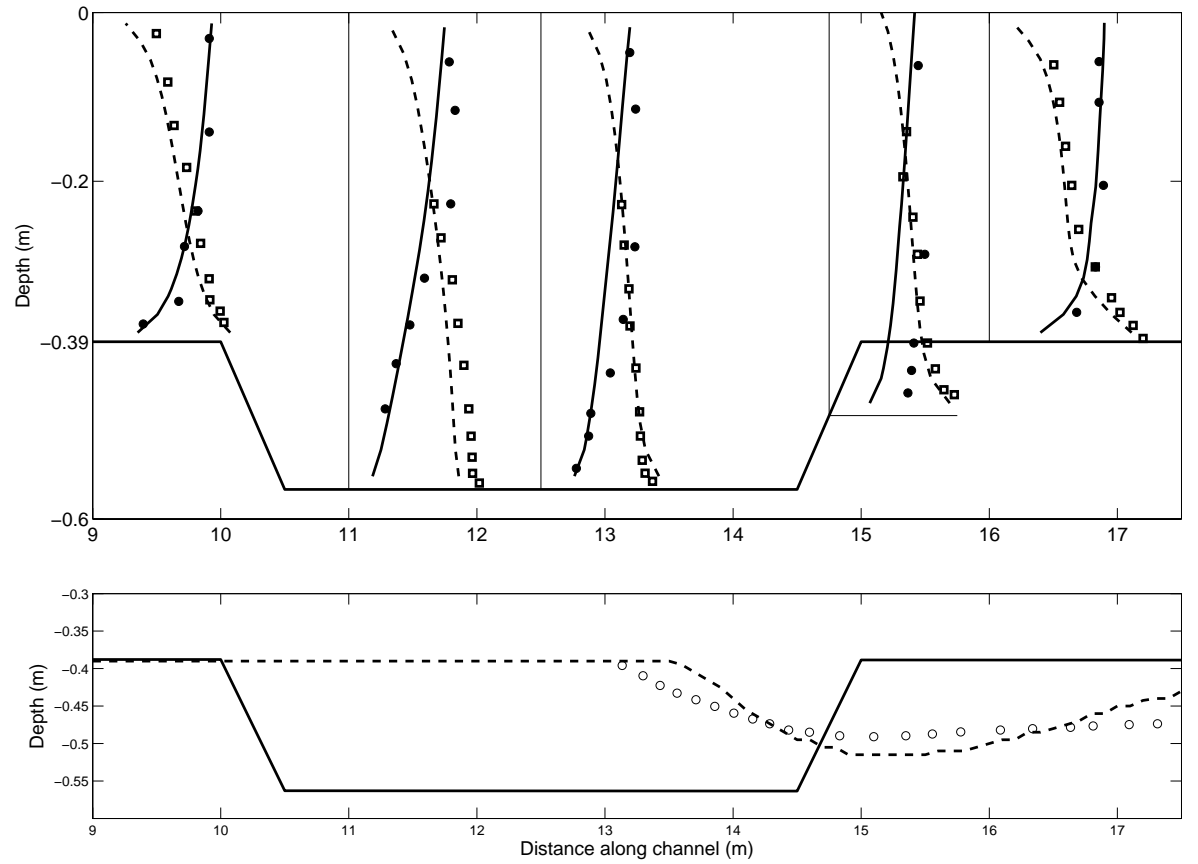

Figure 1. Comparison of velocity and concentration profiles (top) and trench migration (bottom). Top panel: measured (solid circles) and modelled (solid lines) velocities, measured (squares) and modelled (dashed lines) concentrations using a logarithmic scale. Bottom panel: initial (solid line), final measured (circles) and final modelled (dashed line) profiles.
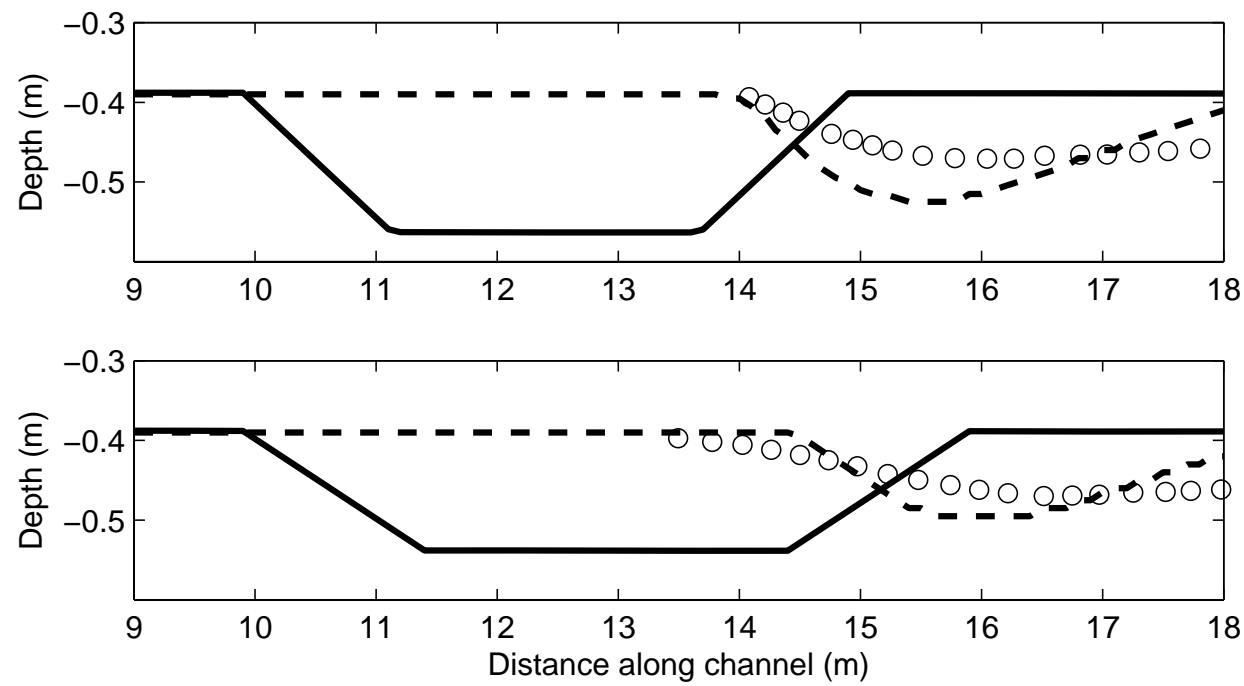

Figure 2. Model-data comparison for the trench migration of trenches of initial slopes 1/7 (top) and 1/10 (bottom). The solid lines represent the initial trench profile, the dashed lines the final numerical profiles and the circles the final experimental profiles. 
Coastal Dynamics 2009

Paper No.

We also use the commonly used Brier Skill Score (BSS) (Sutherland et al., 2004) in order to present a quantitative assessment of the predictive ability of the morphological predictions. The BSS is calculated by comparing the error between the numerical predictions and observations and the error between a baseline prediction and the observations. The baseline prediction is often taken to be the initial morphology, and as such a negative score indicates numerical predictions that would be worse than the initial condition and a perfect score is 1 . The score for the first trench migration (slope of $1 / 3$ ) is found to be 0.94 , which is traditionally considered very good.

In order to further assess the predictive ability of the model rather than its hindcast ability, the migrations of the two other initial trenches (slopes of $1 / 7$ and $1 / 10$ ) were numerically simulated. The sediment and flow conditions were the same experimentally and the model should obtain appropriate predictions using the same parameters. The results are presented in figure 2. Once again, the predictions are reasonable even though the final numerical trenches are found to be a bit deeper than in the experiments. The Brier Skill Score are respectively 0.84 and 0.95 for the $1 / 7$ and 1/10 slopes, again confirming the reasonable to good predictive ability of the model.

Table 3: Summary of the Brier Skill Score for the three initial configurations.

\begin{tabular}{|c|c|}
\hline Initial trench slope & Brier Skill Score \\
\hline $1 / 3$ & 0.94 \\
\hline $1 / 7$ & 0.84 \\
\hline $1 / 10$ & 0.95 \\
\hline
\end{tabular}

\section{Conclusion}

We are developing sediment transport modelling within the framework of a coastal ocean system, which couples a hydrodynamic model, a wave model and an advanced turbulence model. We showed here how sediment transport is being incorporated to the system within the hydrodynamic model. The implementation of the suspended sediment model and of the morphological modelling has been tested using a simple controlled experiment from which both waves and buoyancy are absent. Although the present study is only a first step towards the full realization of sediment transport predictions in real case coastal ocean scenarios, the model-data comparisons did show that the model can correctly predict suspended sediment transport, and morphodynamical evolutions as shown by the Brier Skill Scores obtained and summarized in table 3.

\section{References}

Allen, J, Blackford, J. Holt, J., Proctor, R., Ashworth, M. Siddorn, J. 2001. A highly spatially resolved ecosystem model for the North West European continental Shelf, SARSIA, 86 (6), 423-440.

Canuto V. M., Howard, A., Cheng, Y. and Dubovikov, M. S., 2001. Ocean turbulence. Part I: One-point closure model - Momentum and heat vertical diffusivities, J. Phys. Oceanography, 31: 1413-1426.

Grant, W. D. and O. S. Madsen, 1982. Movable bed roughness in unsteady oscillatory flow, J. Geophys. Res., 87 (C1), 469-481.

Harris, C. K. and Wiberg, P. L., 1997. Approaches to quantifying long-term continental shelf sediment transport with an example from the Northern California STRESS mid-shelf site. Continental Shelf Res., 17 (11), 1389-1418.

Harris, C. K. and Wiberg, P. L., 2001. A two-dimensional, time dependent, model of suspended sediment transport and bed reworking for continental shelves. Computers and Geosciences, 27, 675-690.

Holt, J. T. and James, I. D., 1999. A simulation of the southern North Sea in comparison with measurements from the North Sea Project Part 2 Suspended Particulate Matter, Continental Shelf Res., 19: 1617-1642

Holt, J. T. and James, I. D., 2001. An S coordinate density evolving model of the northwest European continental shelf 1, Model description and density structure. J. Geophys. Res., 106 (C7): 14015-14034.

Holt, J. T. and Umlauf, L., 2008. Modelling the tidal mixing fronts and seasonal stratification of the Northwest European continental shelf, Continental Shelf Res., 28, 887-903.

James, I. D., 1996. Advection schemes for shelf sea models. J. Marine Systems, 8, 237-254.

Killworth, P. D., Stainforth, D., Webb, D. and Paterson, S. M., 1991. The development of a free-surface Bryan-Cox- 
Coastal Dynamics 2009

Paper No.

Semtner ocean model. J. Phys. Oceanography, 21, 1333-1348.

Lesser, G. R., Roelvink, J. A., van Kester, J. and Stelling, G. S., 2004. Development and validation of a threedimensional morphological model, Coastal Eng., 51:883-915.

Malarkey, J. and Davies, A. G., 2003. A non-iterative procedure for the Wiberg and Harris (1994) oscillatory sand ripple predictor. J. Coastal Res., 19 (3), 738-739.

Madsen, O. S., 1994. Spectral wave-current bottom boundary layer flows. Proc. $24^{\text {th }}$ Int. Conf. Coastal Eng., ASCE, 384-398.

Meyer-Peter, E. and Mueller, R., 1948. Formulas for bed load transport. Proc. $2^{\text {nd }}$ IAHR Congress, 39-64.

Nielsen, P., 1992. Coastal bottom boundary layers and sediment transport. World Scientific.

Osuna, P., Wolf, J. and Ashworth, M. I., 2004. Implementation of a wave-current interaction module for the POLCOMS system. Tech. Rep. 168. Proudman Oceanographic Laboratory.

Soulsby, R. L., 1995. Bed shear-stresses due to combined waves and currents. Advances in Coastal Morphodynamics: An Overview of the G8-Coastal Morphdynamics Project, Delft Hydraulics, pp 4.20-4.23.

Soulsby, R.L. and Damgaard, J.S., 2005. Bedload sediment transport in coastal waters. Coastal Eng., 52(8): 673-689.

Sutherland, J., A. H. Peet and R. L. Soulsby, 2004. Evaluating the performance of morphological models. Coastal Eng., 51: 917-939

van Rijn, L. C., 1987. Mathematical Modelling of Morphological Processes in the Case of Suspended Sediment Transport. Delft Hydraulics.

van Rijn, L. C., 1993. Principles of sediment transport in rivers. estuaries and coastal seas. Aqua Publications.

Warner, J. C., Sherwood, C. R., Signell, R. P., Harris, C. K., Arango, H. G., 2008. Development of a three-dimensional, regional, coupled, wave, current and sediment transport model. Computers and Geosciences, 34: 1284-1306.

Wiberg, P. L. and Rubin, D. M., 1989. Bed roughness produced by saltating sediment. J. Geophys. Res., 94 (C4), 50115016.

Wiberg, P. L. and Harris, C. K., 1994. Ripple geometry in wave-dominated environments. J. Geophys. Res., 99 (C1), 775-789 